\title{
Structure and Properties of Tapered Block Polymers of Styrene and Isoprene
}

\author{
Yasuhisa Tsukahara ${ }^{\dagger}$, Norikazu NAKAmura, ${ }^{\dagger \dagger}$ Takeji Hashimoto, \\ and Hiromichi KAWAI
}

Department of Polymer Chemistry, Faculty of Engineering, Kyoto University, Yoshida, Sakyo-ku, Kyoto 606, Japan.

\author{
Tamio Nagaya, Yoshihiro Sugimura, and Shin Tsuge \\ Department of Synthetic Chemistry, Faculty of Engineering, Nagoya University, \\ Furo-cho, Chikusa-ku, Nagoya 464, Japan.
}

(Received February 29, 1980)

\begin{abstract}
Tapered block polymers of styrene and isoprene were prepared by copolymerization of these two monomers, containing a trace amount of THF. $s$-BuLi was used as an initiator, and benzene as the polymerization solvent. Analyses based on pyrolysis gas chromatography and ${ }^{1} \mathrm{H}$ NMR indicated that a considerable amount of mixing of these two monomers took place in the block polymer chain, e.g., for TB-1 to TB-3. Such mixing in the primary structure is considered phenomenologically to give rise to the polystyrene-rich block chain and polyisoprene-rich block chain. The repulsive interaction between the two block chains is much weaker than that between the pure polystyrene and polyisoprene block chains, and this enhances the mixing of the polystyrenerich chain and polyisoprene-rich chain, resulting in a microdomain structure consisting of the polystyrene-rich phase and polyisoprene-rich phase. Owing to this segmental mixing within each phase, the tapered polymer exhibits a lower critical temperature and the unique mechanical properties, in compared to ideal block polymers. The tapered block polymers have the unique solubilizing power of the corresponding homopolymers, arising from their microdomain structures.

KEY WORDS Block Polymer / Polymer Blend / Tapered Block Polymer / Phase Separation / Critical Solution Temperature / Microdomain Structure / Dynamic Mechanical Properties / Partial Mixing /
\end{abstract}

Polymer blends of two incompatible homopolymers undergo phase separation at temperatures below the critical temperature $T_{\mathrm{c}}$ (for systems having upper critical solution temperature $)^{1 \mathrm{a}}$ or above $T_{\mathrm{c}}$ (for systems having lower critical temperature). ${ }^{1 \mathrm{~b}}$ The resulting two-phase structure has a profound influence on the physical properties of the polymer blends. For example, a blend of homopolystyrene (PS) and homopolyisoprene (PI) exhibits the two primary mechanical dispersions due to the onset of microbrownian motions of the PI and PS chains at temperatures very close to those at

\footnotetext{
$\dagger$ Present Address: Department of Synthetic Chemistry, Faculty of Engineering, Nagoya University, Furo-cho, Chikusa-ku, Nagoya 464, Japan.

${ }^{\dagger \dagger}$ Present Address: Tōnan High School, 41, Kisshoinkannodo, Minami-ku, Kyoto 601, Japan.
}

which dispersions for the corresponding homopolymers occur (see for example Figure 9). The modulus at temperatures between the two primarytransition temperatures depends upon the morphology of the phase-separated structure, and especially on the modulus of the continuous phase. ${ }^{2}$

Block polymers composed of the two incompatible polymers, PS and PI also exhibit structureproperty relationships similar to the polymer blends (see for example Figure 10). A slight difference is discernible, however, between the two systems, which is attributed to chain connectivity between PS and PI chains as will be discussed later. The high incompatibility of the constituent polymers (e.g., PS and PI) makes $T_{\mathrm{c}}$ of the corresponding block polymer blends very high. For example, the block polymer TB-6 has a $T_{c}$ of about $360^{\circ} \mathrm{C}$. Such a high 
$T_{\mathrm{c}}$ is unfavorable in consideration of the melt processability, since the phase-separated structure existing below $T_{\mathrm{c}}$ tremendously raises the melt viscosity. ${ }^{3}$ This high incompatibility also causes the mechanical properties of the block polymers to be almost identical to those of the homopolymer blends. This becomes a drawback in a case in which the mechanical properties of the block polymers must be very different from those of the blends.

Such a drawback may be overcome by preparing block polymers having different constituent polymers (with different repulsive interactions), different total molecular weights and different chemical compositions. Generally, $T_{\mathrm{c}}$ decreases with decreasing repulsive interaction of the constituent polymers for a given molecular weight and chemical composition. The temperature $T_{\mathrm{c}}$ also decreases with decreasing molecular weight and with biasing the chemical composition toward 0 or $100 \%$ of the constituent polymers for a given combination of the constituent polymers.

For block polymers having a given combination of constituent polymers (e.g., styrene (S)-isoprene (I) block polymers), total molecular weight and chemical composition, however, this must be overcome by modifying the manner and/or the degree of mixing of the incompatible monomers in the primary structure. As a possible method to modify the primary structure of the S-I block polymers, we shall consider here a simultaneous and livinganionic copolymerization of styrene and isoprene monomers. Such a simultaneous copolymerization generally gives rise to so-called tapered block polymers in which the composition of the isoprene and styrene monomers varies continuously for a given copolymer molecule, e.g., the isoprene fraction varying from almost unity at one end to almost zero at the other end, as has been shown in the works of Hsieh and coworkers, ${ }^{4}$ Zelinski and Childers, ${ }^{5}$ Aggarwal and coworkers, ${ }^{6}$ Kuntz, ${ }^{7}$ Cunningham, and Treiber, ${ }^{8}$ and some others.

In this paper, we study the primary chemical structure of styrene and isoprene tapered block copolymers with pyrolysis gaschromatography and proton NMR, and the morphology of the microphase-separated domain structure with smallangle X-ray scattering (SAXS) and electron microscopy, and dynamic mechanical properties.

\section{POLYMERIZATION}

Isoprene and styrene monomers and the polymerization solvents, tetrahydrofuran (THF) and benzene, were carefully purified by the methods described by Morton et al.,${ }^{9-11}$ and collected under vacuum into respective ampoules equipped with magnetic break-seals.

The tapered block copolymers, TB- 1 to TB- 3 in Table I, were prepared by the simultaneous copolymerization of a mixture of the purified styrene and isoprene monomers, containing a trace amount of THF (about $1 \mathrm{vol}^{\%}$ of the polymerization solvent), with $s$-buthyllithium $(s$-BuLi) in benzene as the polymerization solvent at room temperature.

When a mixture of isoprene and styrene is polymerized in a hydrocarbon solution of an alkyllithium (e.g., benzene solution of $s$-BuLi), the monomer reactivity ratio for isoprenyl anion is much larger than that for styryl anion, ${ }^{4-5}$ causing the propagating polymer chain being rich in isoprene until late in the reaction, whereafter styrene incorporation is suddenly increased. On the other hand, when the mixture is polymerized in a THF solution of $s$-BuLi at $-78^{\circ} \mathrm{C}$ as in the case of TB-5 in table $\mathrm{I}$, the above situation is reversed, i.e., the monomer reactivity ratio for the isoprenyl anion is much smaller than that for the styryl anion, ${ }^{4-5}$ causing the propagating chain to become rich in styrene until late in the reaction; after this, isoprene incorporation is suddenly increased. A trace amount of THF was added in TB-1 to TB-3 so as to reduce blockiness in the sequence distribution of the two monomers or to increase the degree of mixing of the two monomers, i.e., to increase the "taper" character.

The TB- 5 specimen was prepared by polymerizing one half of the styrene monomers at first. After all, the styrene monomers were consumed, a mixture of the other half of the styrene monomers and all the isoprene monomers were simultaneously copolymerized. These polymerizations were carried out with $s$ - BuLi in $\mathrm{THF}$ at $-78^{\circ} \mathrm{C}$. Ideal block polymers, TB- 6 and TB-7 in Table I were prepared by sequential polymerization of styrene monomers first and then the isoprene monomers following consumption of all the styrene monomers with $s$-BuLi in THF at $-78^{\circ} \mathrm{C}$.

Each polymerization was carried out in a solution of about $0.2 \mathrm{moll}^{-1}$ monomer concentration to 
assure a homogeneous reaction, e.g., $11.0 \mathrm{ml}$ for styrene monomer, $14.7 \mathrm{ml}$ for isoprene monomer, and $500 \mathrm{ml}$ for THF or benzene.

In the simultaneous copolymerization process of TB-5 in THF, the reacting medium showed the red color of styryl anion first and then the yellow color of isoprenyl anion, as in the sequential polymerization process in the ideal block of TB-6 and TB-7. This observation indicates that the sequential distribution of the two monomers prepared by the simultaneous copolymerization in THF is very close to that prepared by the sequential polymerization. In contrast to the simultaneous copolymerization in THF, the copolymerization (of TB-1 to TB-3) in benzene in the presence of a trace amount of THF did not exhibit the clear color change but rather exhibited a tenuous reddish yellow through the polymerization reaction, indicating that the copolymerization produces a sequence distribution of the two monomers characteristic for the tapered block copolymer.

The total polymerization time for each sample was kept about $38 \mathrm{~h}$ and termination was carried out by pouring methanol into the polymerization medium. In the case of TB-6 and TB-7, the styrene monomer was first polymerized for $8 \mathrm{~h}$ and then, the isoprene monomer was polymerized for $30 \mathrm{~h}$. For TB-5, the first half of the styrene monomer was polymerized for $8 \mathrm{~h}$ and the mixture of the second half of styrene monomer and all the isoprene monomer was polymerized for $30 \mathrm{~h}$. Following polymerization, each specimen was obtained by precipitating the reacted solution in a large quantity of methanol and then by drying the precipitates in vacuum. Table I summarizes some of the polymerization conditions.

\section{CHARACTERIZATION OF PRIMARY STRUCTURE}

\section{Molecular Weight, Molecular Weight Distribution, Chemical Composition, and Microstructure}

Table I summarizes some of the characteristics of the primary structure of the block polymers. The number-averaged molecular weight was measured by osmometry. All block polymers have narrow molecular weight distribution, and each specimen exhibited a very sharp and single GPC peak. The heterogeneity indices $M_{w} / M_{n}$ determined from GPC are listed also in Table I. The narrow molecular weight distribution of each specimen is also confirmed by a very sharp single peak in the Schlieren pattern of $3-5 \mathrm{gl}^{-1}$ THF solution (observed at $20^{\circ} \mathrm{C}$ in a Sinco Model E ultracentrifuge at 59,780 $\mathrm{rpm})$. The composition of styrene in the block polymer was estimated from elementary analysis and is listed in Table I.

The microstructure of polyisoprene was determined by infrared spectroscopy using the method described in detail in our previous paper. ${ }^{12}$ The tapered block polymers TB-1 to TB-3, polymerized in benzene with a trace amount of THF, have polyisoprene with 1,4-, 1,2-, and 3,4-linkages by 5 , 22 , and $73 \%$, respectively. On the other hand,

Table I. Characterization and polymerization condition of tapered and ideal block polymers of styrene and isoprene

\begin{tabular}{|c|c|c|c|c|c|c|c|}
\hline \multirow{3}{*}{$\begin{array}{l}\text { Specimen } \\
\text { code }\end{array}$} & \multirow{3}{*}{$\begin{array}{c}M_{n} \times 10^{-4 \mathrm{a}} \\
\quad \text { total }\end{array}$} & \multirow{3}{*}{$M_{w} / M_{n}^{\mathrm{b}}$} & \multirow{3}{*}{$\begin{array}{l}\mathrm{wt}^{\circ} \% \text { of }^{\mathrm{c}} \\
\text { styrene }\end{array}$} & \multicolumn{4}{|c|}{ Polymerization condition } \\
\hline & & & & \multirow{2}{*}{ Medium } & Temp & Time & \multirow{2}{*}{ Procedure $^{\mathrm{d}}$} \\
\hline & & & & & ${ }^{\circ} \mathrm{C}$ & $\mathrm{h}$ & \\
\hline TB-1 & 4.3 & 1.05 & 47 & Benzene/THF & 25 & 38 & $\mathrm{~S} / \mathrm{I}(50 / 50)$; tapered \\
\hline TB-2 & 6.3 & 1.08 & 48 & Benzene/THF & 25 & 38 & $\mathrm{~S} / \mathrm{I}(50 / 50)$; tapered \\
\hline TB-3 & 10.6 & 1.09 & 45 & Benzene/THF & 25 & 38 & $\mathrm{~S} / \mathrm{I}(50 / 50)$; tapered \\
\hline TB-5 & 9.7 & 1.18 & 51 & $\mathrm{THF}$ & -78 & $8-30$ & $\mathrm{~S}-\mathrm{S} / \mathrm{I}(25-25 / 50) ;$ tapered \\
\hline TB-6 & 4.9 & 1.13 & 45 & THF & -78 & $8-30$ & $\mathrm{~S}-\mathrm{I}(50-50)$; ideal block \\
\hline TB-7 & 10.2 & 1.18 & 54 & $\mathrm{THF}$ & -78 & $8-30$ & $\mathrm{~S}-\mathrm{I}(50-50)$; ideal block \\
\hline
\end{tabular}


homopolyisoprene, polyisoprene in the tapered polymer of TB-5, and the ideal block polymers of TB-6 and TB-7, polymerized in THF medium, have the microstructures of the respective linkages by 3 , 38 , and $59 \%$.

\section{${ }^{1}$ H NMR Spectra of the Tapered Block Polymers}

Figure 1 illustrates the typical ${ }^{1} \mathrm{H}$ NMR spectra of the tapered block polymers, TB- 2 and TB- 5 , the ideal block polymer TB-6, and the homopolymers, PS and PI. The spectra were measured using about a $5 \%(\mathrm{w} / \mathrm{v})$ polymer solution in carbon tetrachloride with a $60 \mathrm{MHz}{ }^{1} \mathrm{H}$ NMR spectrometer, JNM-RM60 from JEOLCO.

Although TB-1 to TB-3 gave almost identical
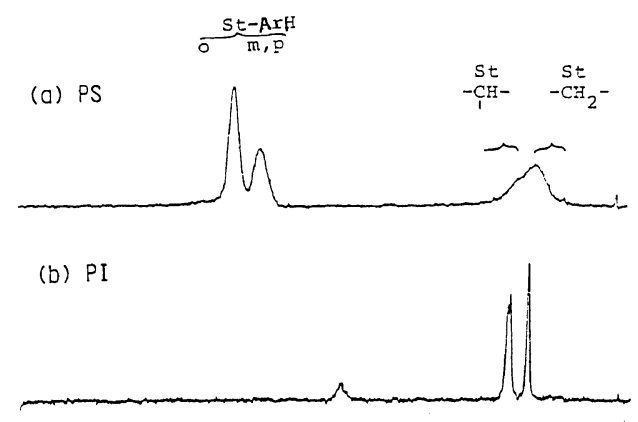

(c) $\mathrm{TB}-5$

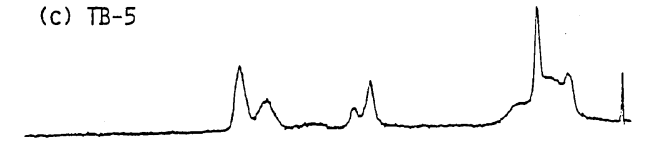

(d) $T B-6$
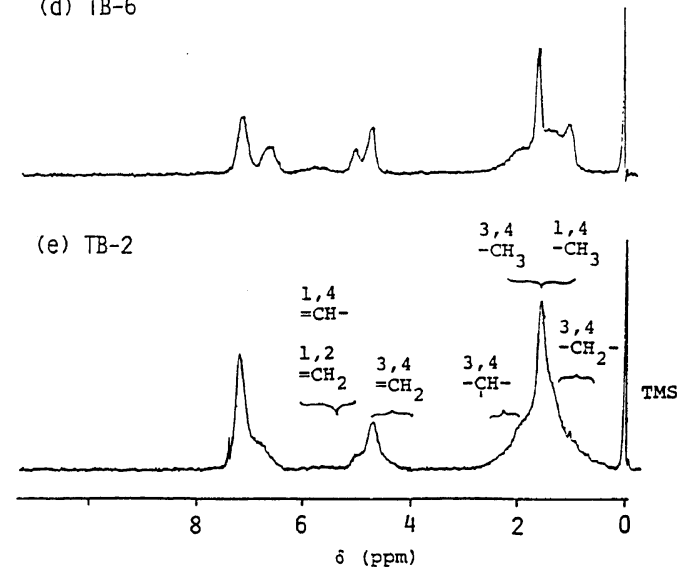

Figure 1. ${ }^{1} \mathrm{H}$ NMR spectra of the tapered block polymers and the associated homopolymers. spectra, fairly big differences can be observed between the spectra for TB-2 and TB-5. The specimens TB-5 and TB-6 (ideal block) give almost identical spectra, suggesting that TB- 5 might be composed of significantly longer sequences of the respective monomer units in comparison with TB-1 to TB-3.

\section{Pyrolysis-Gas Chromatographic Study on the Sequence Distribution in the Tapered Block Polymers}

Figure 2 shows the pyrograms at $520^{\circ} \mathrm{C}$ for the tapered block polymers, the ideal block polymers, the homopolymers and the physical blend. The polymer sample weighing about $200 \mu \mathrm{g}$ was pyrolyzed at $520^{\circ} \mathrm{C}$ under a flow of nitrogen carrier gas in a vertical furnace-type pyrolyzer, Yanagimoto GP$1018^{13-14}$ which was directly attached to a gas chromatograph, the Shimadzu 7-AG having a highresolution glass capillary column (o.d. $0.9 \mathrm{~mm} \times$ i.d. $0.3 \mathrm{~mm} \times 80 \mathrm{~m}$ long) that is suspension-coated with OV-101 and Silanox (325 mesh). The column temperature was programmed from 50 to $250^{\circ} \mathrm{C}$ at a rate of $4^{\circ} \mathrm{C} \mathrm{min}^{-1} .70 \mathrm{ml} \mathrm{min}^{-1}$ of the carrier gas at the pyrolyzer was reduced to $1.0 \mathrm{ml} \mathrm{min}^{-1}$ at the capillary column through a splitter $(70: 1)$. The peak identification was carried out using a directly connected to quadrupole mass spectrometer, JMSQ10A from JEOLCO. The other pyrolysis-gas chromatographic conditions are the same as those described in the previous work. ${ }^{15}$

As can be seen from the pyrograms of PS (a) and PI (b), the former yields the monomer, the dimer and the trimer, and the latter, mostly the monomer and very minute amount of the dimer. There exists a simple additivity for the pyrogram of the physical blend of PS and PI (c). The pyrogram of TB-6 (e) is almost identical to that of the physical blend except for the additional hybrid product of $S I$ (peak d). On the other hand, as might be expected from the ${ }^{1} \mathrm{H}$ NMR, TB-5 and TB-6 give basically the same pyrograms. It is very interesting to notice, however, that TB-5 yields the additonal characteristic peaks which are assigned to SSI or SIS (peak f). Naturally, on the pyrogram of TB-2 (f), a fairly large amount of the hybrid products is observed.

Table II summarizes the fragment distributions on the pyrograms of the tapered polymers. In the table, the values of the estimated minimum junction $\%(M J \%)$ are also listed. $M J \%$ was calculated using 


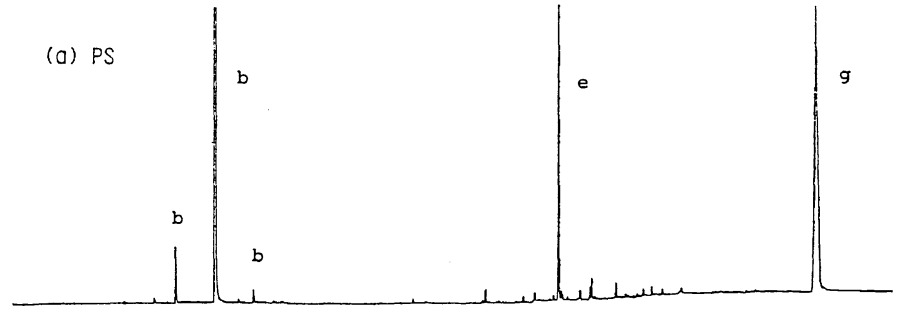

(b) PI

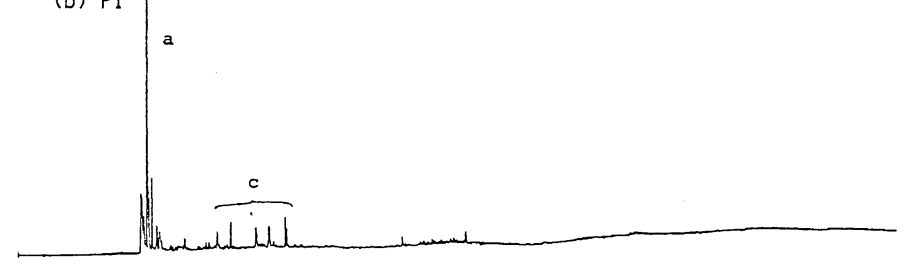

(c) PHYSICAL BLEND (PS=31wt\%)
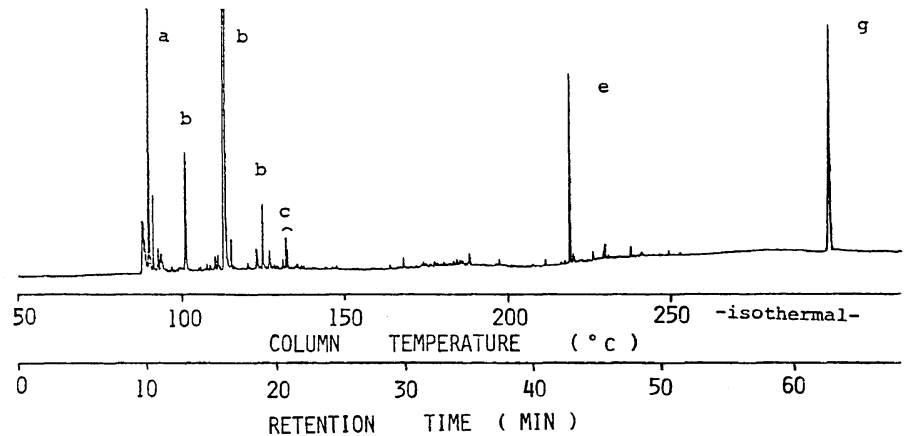

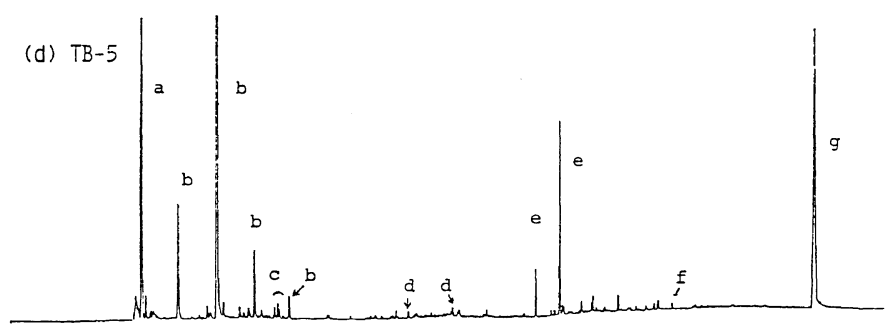

(e) TB-6
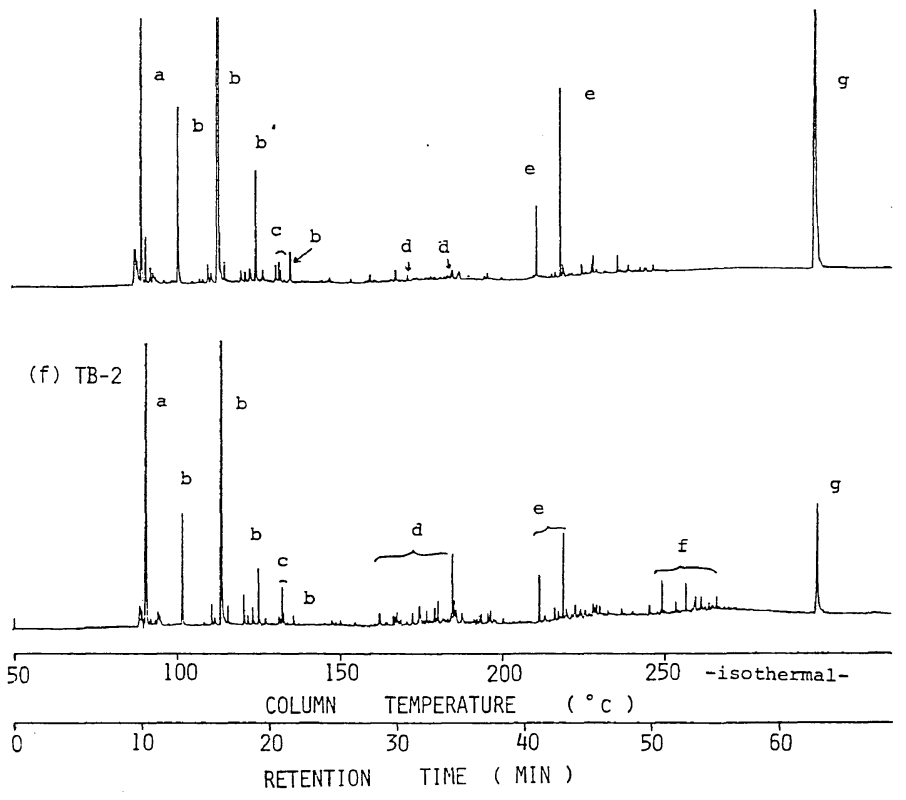

Figure 2. Pyrograms of the tapered block polymers, the associated homopolymers, and the physical blend at the pyrolysis temperature of $520^{\circ} \mathrm{C}: \mathrm{a}, I ; \mathrm{b}, S ; \mathrm{c}, I I ; \mathrm{d}, S I ; \mathrm{f}, S S I+S I S, \mathrm{~g}, S S S$ where $I$ and $S$ are isoprene and styrene units, respectively. 
the data for the hybrid peaks of $\mathrm{d}$ and $\mathrm{f}$, assuming that TB-5 yields the additional characteristic peaks that $\mathrm{f}$ is composed only of the $S S I$ fragment:

$$
M J \%=\frac{1}{2} S I+\frac{1}{3} S S I
$$

where $S I$ and $S S I$ are the relative yields of the hybrid peaks $\mathrm{d}$ and $\mathrm{f}$, listed in Table II. The factors $1 / 2$ and $1 / 3$ are associated with number of junctions per monomer unit for the respective hybrid products.

The estimated $M J \%$ implies that the tapered polymers TB-1 to TB-3 should have at least the same amount of the junctions $\sim S I \sim$ along the molecular chain. The value for TB-6 $(0.17 \%)$ is very close to the theoretical junction $\%$ of 0.18 calculated from the molecular weight of 49,000 . The data for TB-1 to TB-3 suggest that these tapered polymers should have more than 4 junctions within 100 monomer units along the molecular chain; that is, the average sequence length should be less than 25 monomer units. On the other hand, a comparison of the data for TB- 5 and TB- 6 suggest that the tapered polymer TB-5 should incorporate the junction about 1.4 times those for the ideal block polymer TB-6.

As was discussed in the previous paper, ${ }^{16,17}$ the relative styrene monomer yield, $Y(S)_{\text {rel }}$ could be used as an index of the randomness of the monomer arrangement because of the boundary monomer effect on the formation of the degradation products. Here, since PS degrades mostly into the monomer, the dimer and the trimer, the $Y(S)_{\text {rel }}$ is defined as follows:

$$
Y(S)_{\mathrm{rel}}=\frac{Y(S)}{Y(S)+Y(S S)+Y(S S S)} \times 100
$$

where $Y(S), Y(S S)$, and $Y(S S S)$ are the yields of the monomer, the dimer and the trimer, respectively. The calculated $Y(S)_{\text {rel }}$ for the tapered polymers are also listed in Table II. These data also suggest that the styrene monomer units in TB- 1 to TB-3 are more finely neighbored by the counter monomer (isoprene) unit than those in TB-5 whereas the $Y(S)_{\text {rel }}$ value of TB-5 is the same as that of TB-6.

\section{SUPERMOLECULAR STRUCTURE}

The film specimens of the block polymers were prepared by casting about $10 \%$ toluene solutions onto the glass plate. The specimens were further dried under a vacuum for a few weeks until the films were at constant weight. The microphase-separated structure of the block polymers, if any, were investigated by electron microscopy and the smallangle X-ray scattering (SAXS) technique.

\section{Electron Microscopy}

For making electron microscopic observations, the specimens were stained in an aqueous solution of osmium tetroxide for $24 \mathrm{~h}$ at room temperature and subsequently cut into ribbon shape, embedded in epoxy resin, trimmed, and stained again in the aqueous solution of $\mathrm{OsO}_{4}$ for $5-12 \mathrm{~h}$ at $50^{\circ} \mathrm{C}$ and then cut normally to the film surface by a LKB

Table II. Fragment distributions from tapered block polymers

\begin{tabular}{|c|c|c|c|c|c|c|c|c|c|}
\hline \multirow{3}{*}{$\begin{array}{l}\text { Specimen } \\
\text { code }\end{array}$} & \multicolumn{7}{|c|}{ Fragment distribution (monomer unit ratio $\%)^{a}$} & \multirow{3}{*}{$\frac{Y(S)_{\mathrm{rel}}^{\mathrm{b}}}{\%}$} & \multirow{3}{*}{$\begin{array}{c}\begin{array}{c}\text { Estimated } \\
\text { minimum } \\
\text { junctions }\end{array} \\
\% \\
\%\end{array}$} \\
\hline & \multirow{2}{*}{$I(\mathrm{a})$} & \multirow{2}{*}{$S(\mathrm{~b})$} & \multirow{2}{*}{$I I(\mathrm{c})$} & \multirow{2}{*}{$S I(\mathrm{~d})$} & \multirow{2}{*}{$S S(\mathrm{e})$} & \multirow{2}{*}{$S S I+S I S(\mathrm{f})$} & \multirow{2}{*}{$S S S(\mathrm{~g})$} & & \\
\hline & & & & & & & & & \\
\hline TB-1 & 23.0 & 57.5 & 1.9 & 5.7 & 4.0 & 3.4 & 4.6 & 88.6 & 4.0 \\
\hline TB-2 & 26.1 & 55.0 & 2.6 & 5.9 & 3.3 & 3.1 & 4.0 & 88.3 & 4.0 \\
\hline TB-3 & 28.1 & 54.8 & 2.2 & 5.5 & 3.3 & 2.8 & 3.3 & 89.2 & 3.7 \\
\hline TB-5 & 18.6 & 67.7 & 1.2 & 0.33 & 3.0 & 0.23 & 9.0 & 84.9 & 0.24 \\
\hline TB-6 & 18.0 & 68.2 & 1.3 & 0.34 & 2.9 & - & 9.2 & 84.9 & 0.17 \\
\hline
\end{tabular}
and estimated minimum junction $\%$

a (a) - (g) correspond to the peak numbers on Figure 2.

b Relative styrene monomer yield calculated from eq 1 .

c $M J \%$ calculated by $(S I / 2+S S I / 3)$. 
ultramicrotome to a thickness of about $30 \mathrm{~nm}$. It is well known that osmium tetroxide selectively stains the polyisoprene phase and not the polystyrene phase, giving rise to a deep contrast in the image so that the stained and unstained phases appear dark and bright, respectively.

Figures 3 and 5 show typical electron micrographs for the tapered block copolymers of TB-1 to TB-3, respectively, and Figure 6 shows the one for the tapered block copolymer TB-5. On the other hand, Figure 7 represents the micrographs for the ideal block polymers of (a) TB-6 and (b) TB-7.

The general trend is that the tapered block polymers have microdomain structures similar to those of ideal block polymers. However, the image contrast of the two phases for TB-1 to TB-3 is much weaker than that for the ideal block polymers TB-6 and TB-7. In contrast to TB-1 to TB-3, the image contrast for the tapered polymer TB- 5 is almost identical to and as strong as that for the ideal block polymers.

It is a well accepted fact that the polystyrene (polyisoprene) domains in the ideal block polymers are composed almost solely of the polystyrene (polyisoprene) block chains ${ }^{18}$ due to repulsive interactions between the two components, and that very little mixing occurs except in the interfacial regions..$^{23-25}$ The fact that the contrast is very weak for the TB- 1 to TB-3 suggests the existence of a

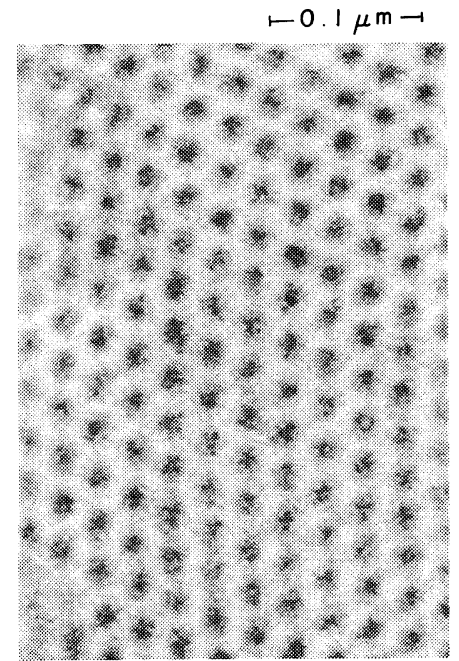

Figure 3. Typical transmission electron micrograph of the tapered block polymer TB-1 cast from toluene solution; stained with $\mathrm{OsO}_{4}$ aqueous solution.

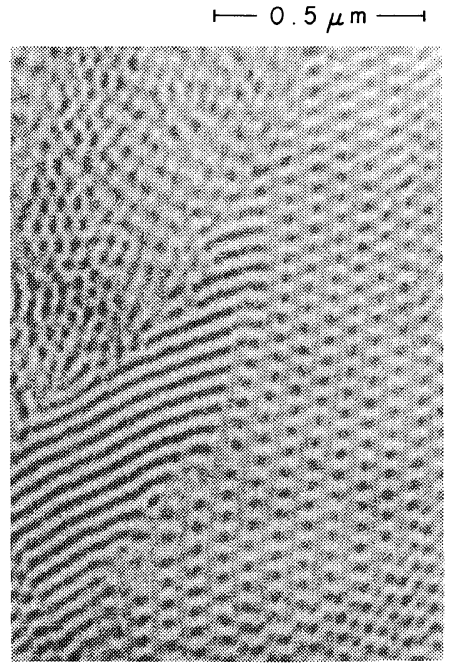

Figure 4. Typical transmission electron micrograph of the tapered block polymer TB-2 cast from toluene solution; stained with $\mathrm{OsO}_{4}$ aqueous solution.

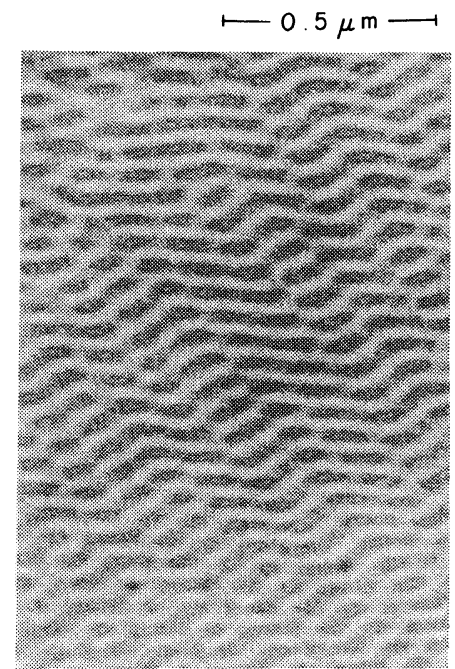

Figure 5. Typical transmission electron micrograph of the tapered block polymer TB-3 cast from toluene solution; stained with $\mathrm{OsO}_{4}$ aqueous solution.

substantial mixing of the incompatible components within each phase.

Namely, the tapered block polymers of TB-1 and TB-3 may be simplified as being composed of, at least as a first approximation, the "polystyrene-rich 


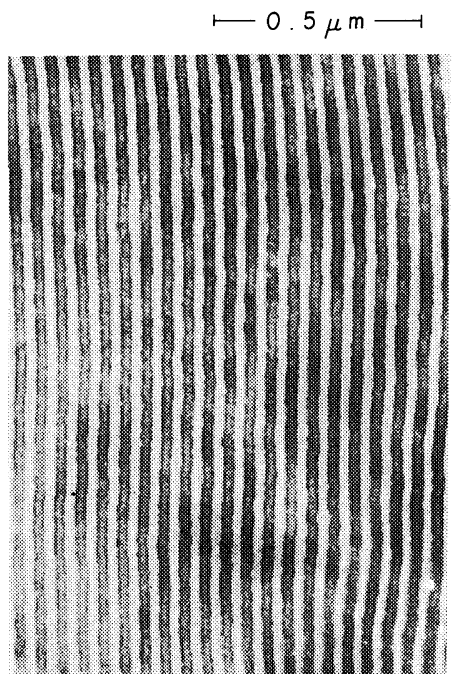

Figure 6. Typical transmission electron micrograph of the tapered block polymer TB-5 cast from toluene solution; stained with $\mathrm{OsO}_{4}$ aqueous solution.

block chain" and "polyisoprene-rich block chain," corresponding to pure polystyrene and polyisoprene block chains in the ideal block polymers. The mixing of the incompatible components in each block chain should decrease the repulsive interactions between the polystyrene-rich block chains and the polyisoprene-rich block chain, thus resulting in an enchanced mixing of the two types of block chains within each phase. Therefore, the dark and bright phases in TB-1 to TB-3 tapered polymers correspond to "polyisoprene-rich domains" and "polystyrene-rich domains," respectively.

In contrast to the TB-1 to TB-3, the tapered polymer of TB-5 undergoes a much smaller degree of mixing of the incompatible component in each block chain as is clarified from the pyrolysis gas chromatography, ${ }^{1} \mathrm{H}$ NMR spectroscopy. Consequently, the repulsive interaction between the two types of the block chains is not much different from that of the ideal block, so that each domain is composed almost completely of its own component as in the case of ideal block polymers. ${ }^{24}$

The ideal block polymers, TB- 6 and TB-7, and the tapered block polymer TB-5 have almost equal fractions of styrene and isoprene, and therefore nearly equal molecular weights for the two block sequences due to the very small degree of mixing of the two components in each block chain.

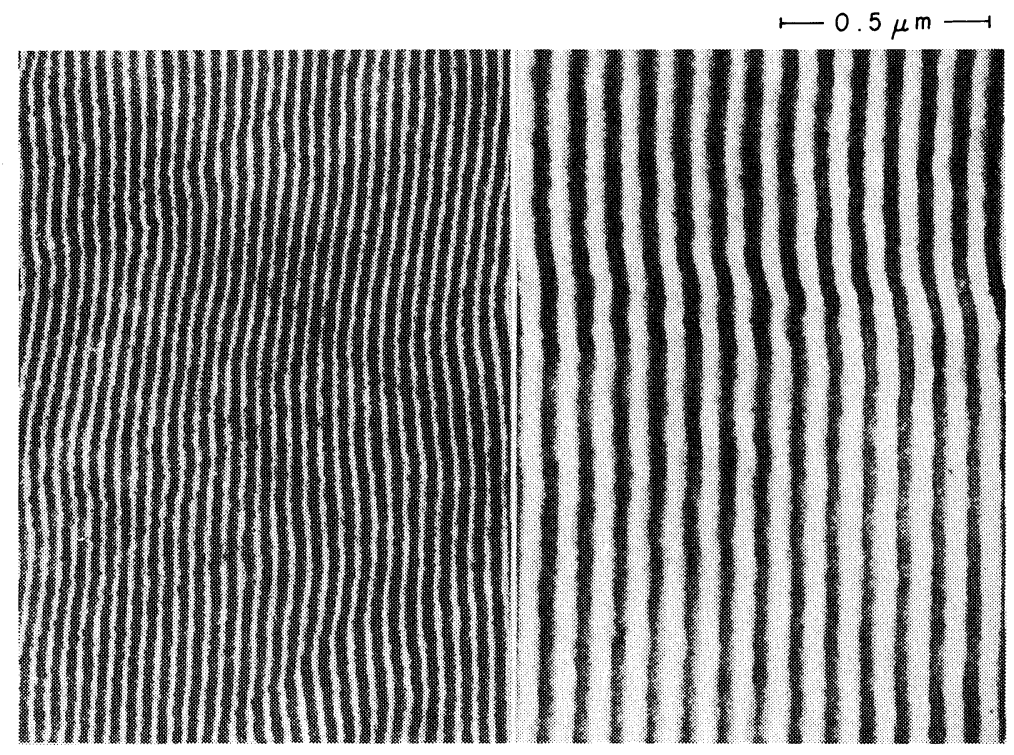

(a)

(b)

Figure 7. Typical transmission electron micrograph of the ideal block polymers, (a) TB-6 and (b) TB-7, cast from toluene solutions; stained with $\mathrm{OsO}_{4}$ aqueous solution. 
Consequently, these specimens exhibit regular alternating lamellar microdomains.

The morphology of the tapered polymers TB-1 to TB-3 is more complicated than that for TB-5 to TB7 , although the fraction of the contituent monomeric units is kept almost identical for these polymers. The specimen TB-1 exhibits, more or less, a rod-like domain morphology in which polyisoprene-rich rods are dispersed in the polystyrene-rich matrix (Figure 3). The rod morphology of TB-1 can be further confirmed by the SAXS pattern as will be described eleswhere. ${ }^{27}$ This evidence that TB-1 exhibits a rod-like morphology rather than the lamellar morphology may be phenomenologically interpreted on the basis of a sequence distribution of the two monomers in a block polymer chain, making the polyisoprene-rich block chain shorter than the polystyrene-rich block chain.

The specimens TB-2 and TB-3 exhibit rod-like or lamella-like morphology as shown in Figures 3 and 4, respectively. Each micrograph shows Moiré patterns which have never before observed in cylindrical or lamellar microdomain morphology in the ideal block polymer systems. The Moiré pattern seems to suggest a uniplanar arrangement of the rods or lamellae with misaligned orientation of their axes in the successive planes.

\section{SAXS Analysis}

The SAXS curves from the ideal block polymers and tapered.block polymer TB- 5 are described in detail in our previous paper ${ }^{24}$ (TB-5-TB-7 corresponding to L-5, L-3, and L-6 specimens, respectively, and the respective long identity period being 46,32 , and $50 \mathrm{~nm}$ ).

As discussed earlier, the mixing of the incompatible components in the block chain decreases the repulsive interactions between the polystyrene-rich and polyisoprene-rich block chains. The reduced interactions should then cause the critical temperature $T_{\mathrm{c}}$ to decrease. Thus, the tapered polymer should have a lower $T_{\mathrm{c}}$ than the ideal block polymer of corresponding molecular weight and chemical composition. This is confirmed by the experimental results shown in Figure 8 where changes in the scattering curves with elevating temperature were investigated for (a) TB-1 and (b) TB-6. The two specimens have nearly equal molecular weights, and the same chemical composition as shown in Table I.

The SAXS curves were measured with a position sensitive X-ray detector and $12 \mathrm{~kW}$ rotating anode $\mathrm{X}$-ray generator. ${ }^{19}$ In the oscilloscope displays, the origin of each curve was shifted along the diagonal direction to avoid overlaps. Each curve was obtained with $40 \mathrm{~s}$ exposure to X-ray, each channel corresponds to one minute in the scattering angle

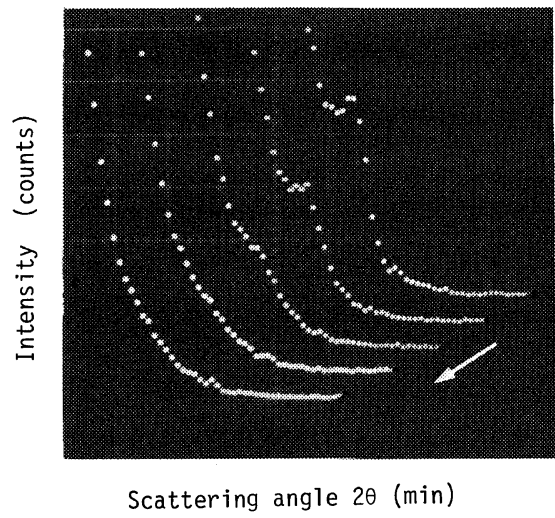

(a)

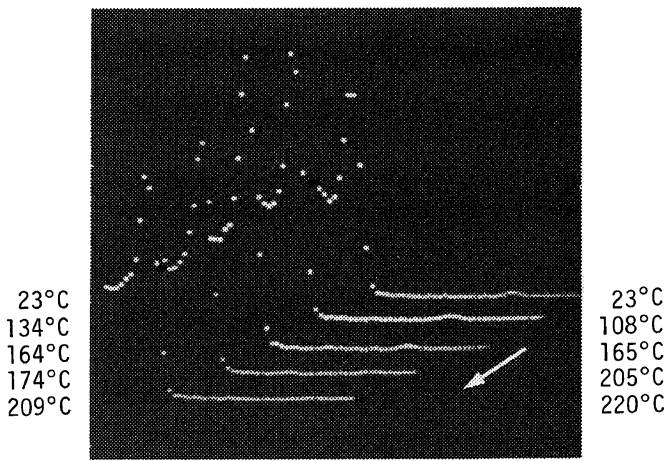

Scattering angle $2 \theta(\min )$

(b)

Figure 8. Change of the SAXS curves with elevating temperature for (a) the tapered block polymer TB-1 and (b) the ideal block polymer TB-6. Each curve was measured with $40 \mathrm{~s}$ exposure to X-ray and shifted along the diagonal direction to avoid an overlap of each curve. One channel corresponds to one minute in scattering angle $(\mathrm{Cu}-\mathrm{K} \alpha$ radiation). 
( $\mathrm{Cu}-\mathrm{K} \alpha$ radiation). It should be noted that the scattering maximum corresponding to the long identity period disappears with elevating temperature at around $170^{\circ} \mathrm{C}$ as a consequence of the melting of the microphase-separated domains into a homogeneous mixture. ${ }^{28}$ The critical temperature of $170^{\circ} \mathrm{C}$ is shown to be much lower than that of the ideal block polymer for which the temperature is observed higher than $220^{\circ} \mathrm{C}$, at least (being expected to be $360^{\circ} \mathrm{C}$ from Helfand's theor $\mathrm{y}^{20}$ ).

\section{DYNAMIC MECHANICAL PROPERTIES}

We shall study here the effect of the tapered monomer distribution on dynamic mechanical properties in comparison of those of the mechanical blend and ideal block polymers.

Figure 9 shows isochronal dynamic shear moduli $G^{\prime}$ and $G^{\prime \prime}$ at $c a .0 .1 \mathrm{~Hz}$ measured by torsion pendulum method for PS and PI and their mechanical mixture having a composition of $50 \mathrm{wt} \%$ PS. The

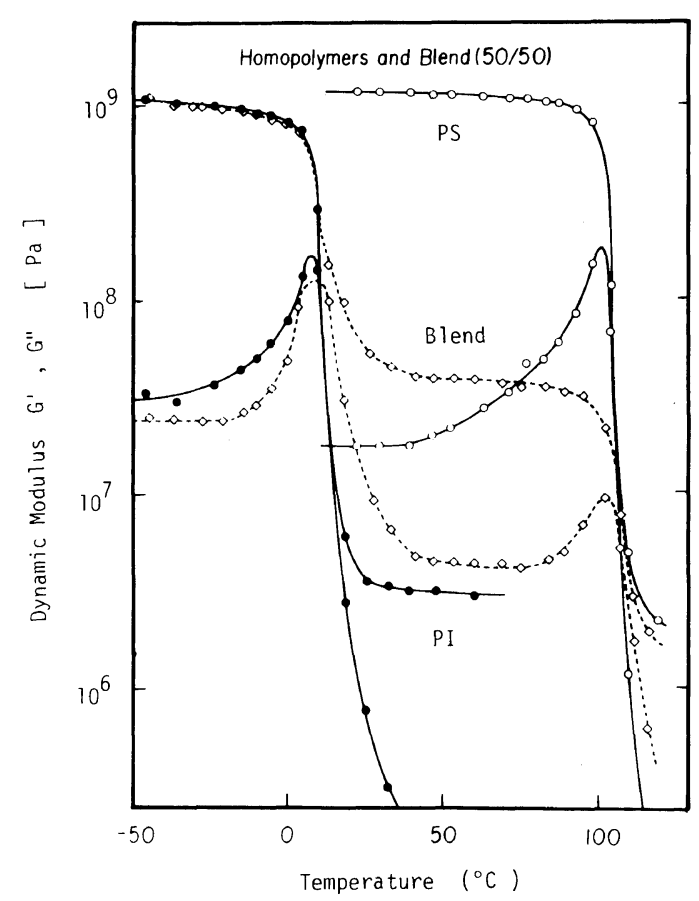

Figure 9. The isochronal shear moduli $G^{\prime}$ and $G^{\prime \prime}$ for homopolystyrene (PS) and homopolyisoprene (PI) (solid lines) and their mechanical mixture (broken lines) at $c a$. $0.1 \mathrm{~Hz}$.
PS and PI specimens have number-average molecular weights of 170 and $1,120 \mathrm{~kg} \mathrm{~mol}^{-1}$, respectively. The blend exhibits the two mechanical dispersions arising from the onset of the microbrownian motions of PI and PS segments in the respective phase-separated domains. The temperature of each primary dispersion in the blend is almost identical to that of the respective homopolymer. It should be noted that the PI and also the polyisoprene block sequence in the block polymers have a high vinyl content resulting in the corresponding primary dispersion occuring at $17^{\circ} \mathrm{C}$ at $0.1 \mathrm{~Hz}$.

Figure 10 shows isochronal dynamic tensile moduli $E^{\prime}$ and $E^{\prime \prime}$ and the loss tangent at $10 \mathrm{~Hz}$ for tapered polymer TB-1, ideal block polymer TB-6, and a mechanical blend of PS and PI. The molecular weights of PS and PI are 277 and $490 \mathrm{~kg} \mathrm{~mol}^{-1}$,

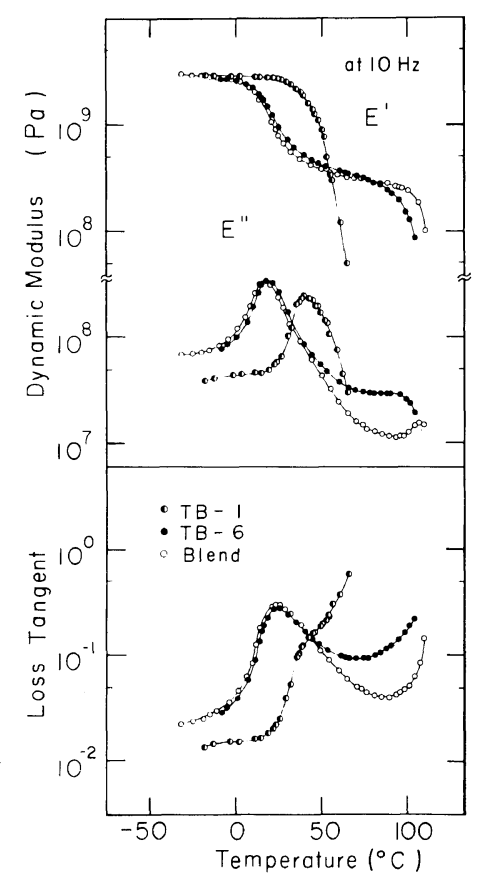

Figure 10. The isochronal dynamic tensile moduli $E^{\prime}$ and $E^{\prime \prime}$ and loss tangent at $10 \mathrm{~Hz}$ for the tapered block polymer TB-1, the ideal block polymer TB-6, and a mechanical mixture of polystyrene (PS) and polyisoprene (PI). Molecular weights of PS and PI are 277 and 490 $\mathrm{kg} \mathrm{mol}^{-1}$, respectively, and they are mixed by equal weight. 
respectively, and equal weights of each are mixed. The ideal block polymer exhibits the dynamic mechanical properties quite similar to those of the blend. However, subtle differences are discernible between the two systems: (i) the glass-transition temperature of the polystyrene segments in the block polymer is slightly lower than that in the blend or in the homo-PS, and (ii) $E^{\prime}$ of the block polymer decreases with temperature (between the two glass-transition temperatures) much faster than the blend, or $E^{\prime \prime}$ and $\tan \delta$ of the block polymers are larger than those of the blend in the corresponding temperature range.

The differences as described above should be attributed to the chain connectivity between PS and PI chains. That is, the chain connnection in the block polymer limits the phase separation to a submicroscopic scale resulting in the microphase separation. The relationship between the molecular dimension and the size of the microphase-separated domain has already been clarified in some of our earlier works. ${ }^{21-25}$ Due to this microphase separation, the block polymer systems generally have an extraordinary large interfacial area and interfacial volume as compared to the polymer blend systems, and this results in enchanced mechanical interactions between the two phases. An another effect of the chain connectivity on the mechanical properties may be visualized in terms of the cooperativity of the molecular dynamics. That is, the molecular motions of the polyisoprene generally affect some of the molecular motions of polystyrene, thus causing an excess free volume in the polystyrene phase. The cooperativity of the molecular motions should be higher in block polymer systems than in the blend systems.

Owing to the extensive mixing of the two incompatible components in the block chains and therefore in each phase, the dynamic mechanical properties of the TB-1 specimen are drastically different from those of the blend and ideal block polymers in that they show only one distinctive viscoelastic dispersion maximum at $41^{\circ} \mathrm{C}$ (plus a faint shoulder at around $55^{\circ} \mathrm{C}$ ) in contrast to the polymer blend and ideal block polymers which exhibit the two dispersion maxima (at 17 and $107^{\circ} \mathrm{C}$ and at 18 and $96^{\circ} \mathrm{C}$, respectively). The occurrence of a single dispersion maximum between the two primary transition temperatures is somewhat similar to the random copolymers or alternating copolymers.
A distinctive feature of the tapered block polymers in comparison to the random or alternating copolymers, however, is the solubilizing power of the homopolymers. That is, the tapered block polymers can solubilize the respective homopolymers provided the molecular weight of the homopolymers is nearly equal to or less than that of the corresponding block sequences as in the case of the ideal block polymers. ${ }^{26}$ Thus, if homopolyisoprene, having a molecular weight less than or equal to that of polyisoprene-rich block chain of a tapered polymer, is added to the tapered polymer, a large fraction of the polyisoprene is solubilized into the polyisoprene-rich domains and a minor fraction may be solubilized even into the polystyrene-rich domains. Thus, the solubilized homopolyisoprene should generally affect the primary transition of the polystyrene-rich phase as well as that of the polyisoprene-rich phase.

The speculation drawn above is clearly confirmed by the preliminary results shown in Figure 11 which shows the isochronal dynamic mechanical properties of the tapered polymer TB-4 and its

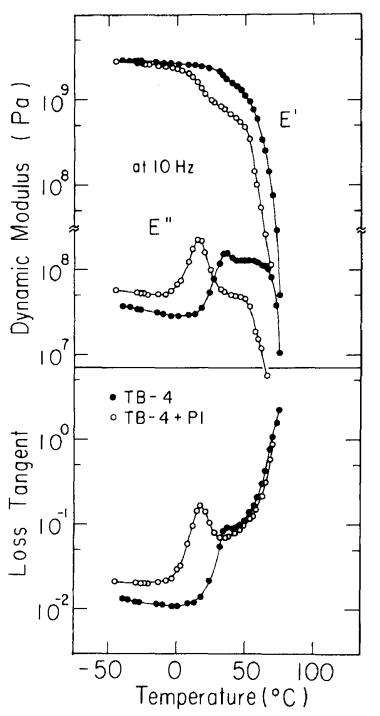

Figure 11. The isochronal dynamic tensile moduli $E^{\prime}$ and $E^{\prime \prime}$ and loss tangent at $10 \mathrm{~Hz}$ for the tapered polymer TB-4 and its blend with homopolyisoprene (PI). The TB4 has total molecular weight of $345 \mathrm{~kg} \mathrm{~mol}^{-1}$, weight fraction of styrene of 0.51 and are polymerized with the same method as for TB-1 and TB-3. The PI has molecular weight of $490 \mathrm{~kg} \mathrm{~mol}^{-1}$. 
blend with homopolyisoprene. The TB-4 has total molecular weight of $345 \mathrm{~kg} \mathrm{~mol}^{-1}$, and the weight fraction of styrene is 0.51 . It is polymerized with the same method used for TB-1 to TB-3. The homopolyisoprene has molecular weight of $490 \mathrm{~kg} \mathrm{~mol}^{-1}$. It can be clearly seen that the homopolyisoprene lowers the transition temperature of the polyisoprene-rich phase from $37^{\circ} \mathrm{C}$ to $17^{\circ} \mathrm{C}$ and that of the polystyrene-rich phase from $62^{\circ} \mathrm{C}$ to $38^{\circ} \mathrm{C}$. The lowering of the two transition temperatures should be best interpreted in terms of the solubilization of the homopolymers into each phase.

The degree of mixing of the polystyrene-rich segment into the polyisoprene-rich phase or the polyisoprene-rich segment into the polystyrene-rich phase is generally a function of the molecular weight. The larger the molecular weight, the less the degree of mixing, so that the mechanical properties are closer to those characteristic of the two-phase structure. The difference in the mechanical properties of TB-1 and TB-4 may be considered to arise from the molecular weight dependence of the degree of the mixing on the incompatible block chains.

Acknowledgements. Kyoto University group acknowledges the support provided by a Grant-in-Aid for Scientific Research from the Ministry of Education, Science and Culture, Japan (No. 243021) and that from a scientific research Grant provided by the Japan Synthetic Rubber Co., Ltd., Tokyo, Japan, and the Bridge Stone Tire Co., Ltd., Tokyo, Japan.

\section{REFERENCES}

1. (a) R. S. Scott, J. Chem. Phys., 17, 279 (1949). (b) See, for example, I. C. Sanchez, in "Polymer Blends," Vol. I, D. R. Paul and S. Newman Ed., Academic Press, New York, N.Y., 1978, Chapter 3.

2. K. Fujino, Y. Ogawa, and H. Kawai, J. Appl. Polym. Sci., 8, 2147 (1964).

3. E. Gouinlock and R. S. Porter, Polym. Eng. Sci., 17, 535 (1977).

4. H. L. Hsieh, in "Block and Graft Copolymers," J. J. Burke and V. Weiss, Ed., Syracuse University Press, Syracuse, N.Y. (1973).
5. R. Zelinski and C. W. Childers, Rubber Chem. Technol., 41, 161 (1968).

6. S. L. Aggarwal, R. A. Livigni, L. F. Marker, and T. J. Duclek, in "Block and Graft Copolymers," J. J. Burke and V. Weiss, Ed., Syracuse University Press, Syracuse, N.Y. (1973).

7. I. Kuntz, J. Polym. Sci., 54, 569 (1961).

8. R. F. Cunningham and M. R. Treiber, J. Appl. Polym. Sci., 12, 23 (1968).

9. M. Morton, A. A. Rembaum, and J. L. Hall, $J$. Polym. Sci., A, 1, 361 (1963).

10. M. Morton and R. Milkvich, J. Polym. Sci., A, 1, 443 (1963).

11. M. Morton, E. E. Bostick, and R. G. Clarke, J. Polym. Sci., A, 1, (1963).

12. T. Hashimoto, N. Nakamura, M. Shibayama, A. Izumi, and H. Kawai, J. Macromol. Sci., Phys., in press.

13. S. Tsuge and T. Takeuchi, Anal. Chem., 49, 348 (1977).

14. Y. Sugimura and S. Tsuge, Anal. Chem., 50, 1968 (1978).

15. S. Tsuge, T. Kobayashi, T. Nagaya, and T. Takeuchi, Macromolecules, 12, 988 (1979).

16. S. Tsuge, T. Kobayashi, T. Nagaya, and T. Takeuchi, J. Appl. Anal. Pyrolysis, 1, 133 (1979).

17. T. Nagaya, Y. Sugimura, and S. Tsuge, Macromolecules, in press.

18. H. Kim, Macromolecules, 5, 594 (1972).

19. partially described by M. Shibayama, M. Fujimura, K. Saijio, S. Suehiro, T. Hashimoto, and H. Kawai, Polym. Prepr. Jpn., 27, 1652 (1978). T. Hashimoto, S. Suehiro, M. Shibayama, M. Fujimura, K. Saijio and H. Kawai, to be submitted to Polym. J.

20. E. Helfand and Z. R. Wasserman, Macromolecules, 9, 879 (1976).

21. T. Inoue, T. Soen, T. Hashimoto, and H. Kawai, $J$. Polym. Sci., A-2, 7, 1283 (1969).

22. T. Soen, T. Inoue, K. Miyoshi, and H. Kawai, $J$. Polym. Sci., A-2, 10, 1757 (1972).

23. T. Hashimoto, A. Todo, H. Itoi, and H. Kawai, Macromolecules, 10, 377 (1977).

24. T. Hashimoto, M. Shibayama, and H. Kawai, Macromolecules, in press.

25. T. Hashimoto, M. Fujimura, and H. Kawai, submitted to Macromolecules.

26. T. Inoue, T. Soen, T. Hashimoto, and H. Kawai, Macromolecules, 3, 87 (1970).

27. Y. Tsukahara, T. Hashimoto, and H. Kawai, to be published.

28. T. Hashimoto, Y. Tsukahara, and H. Kawai, $J$. Polym. Sci., Polym. Lett., Ed., in press. 